\title{
A Novel p.Glu298Lys Mutation in the ACMSD Gene in Sporadic Parkinson's Disease
}

Dolores Vilas ${ }^{\mathrm{a}}$, Rubén Fernández-Santiago ${ }^{\mathrm{b}, \mathrm{c}, \mathrm{d}}$, Elena Sanchez ${ }^{\mathrm{e}}$, Luis J. Azcona ${ }^{\mathrm{e}, \mathrm{f}}$, Meritxell Santos-Montes ${ }^{\mathrm{a}}$, Pilar Casquero ${ }^{\mathrm{g}}$, Lucía Argandoña ${ }^{\mathrm{g}}$, Eduardo Tolosa ${ }^{\mathrm{a}, \mathrm{c}, \mathrm{d}, 1, *}$ and Coro Paisán-Ruiz ${ }^{\mathrm{e}, \mathrm{h}, \mathrm{i}, 1, *}$

${ }^{a}$ Movement Disorders Unit, Neurology Service, Hospital Clínic de Barcelona, Barcelona, Spain

${ }^{\mathrm{b}}$ Department of Neurology, Laboratory of Neurodegenerative Disorders, Hospital Clínic de Barcelona, Barcelona, Spain

${ }^{\mathrm{c}}$ Institut d'Investigacions Biomèdiques August Pi i Sunyer (IDIBAPS), University of Barcelona, Barcelona, Spain

${ }^{\mathrm{d}}$ Centre for Networked Biomedical Research on Neurodegenerative Diseases (CIBERNED), Spain

${ }^{\mathrm{e}}$ Department of Neurology, Icahn School of Medicine at Mount Sinai, One Gustave L. Levy Place, New York, $N Y, U S A$

${ }_{\mathrm{f}}^{\mathrm{f}}$ Department of Neurosciences, Icahn School of Medicine at Mount Sinai, One Gustave L. Levy Place, New York, $N Y, U S A$

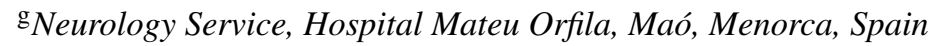

${ }^{\mathrm{h}}$ Departments of Psychiatry and Genetics and Genomic Sciences, Icahn School of Medicine at Mount Sinai, One Gustave L. Levy Place, New York, NY, USA

${ }^{\mathrm{i}}$ Friedman Brain and Mindich Child Health and Development Institutes, Icahn School of Medicine at Mount Sinai, One Gustave L. Levy Place, New York, NY, USA

Accepted 11 June 2017

\begin{abstract}
.
Background: Common genetic variability in the $A C M S D$ gene has been associated with increased risk for Parkinson's disease (PD) but $A C M S D$ mutations in clinical cases of PD have so far not been reported.

Objective: To describe a case of sporadic PD carrying a novel ACMSD mutation.

Methods: As part of a genetic study to identify potential pathogenic gene defects related to PD in the Mediterranean island Menorca, an initial group of 62 PD patients underwent mutational screening using a panel-based sequencing approach.

Results: We report a 74-years-old man with sporadic PD who developed tremor in his right hand and slowness. On examination, moderate rigidity, asymmetric bradykinesia, and bilateral action tremor were present. He was started on levodopa with significant improvement. Two years later, he developed wearing off phenomena. The genetic study in the patient identified a novel ACMSD mutation resulting in p.Glu298Lys amino-acid change which was not present in neurologically normal population. Conclusions: Our data suggest that not only common genetic variability but also rare variants in $A C M S D$ alone or in combination with other risk factors might increase the risk of PD.
\end{abstract}

Keywords: ACMSD, Parkinson's, genetic, kynurenine

\footnotetext{
${ }^{1}$ These authors contributed equally to this work.

* Correspondence to: Eduardo Tolosa, Neurology Service, Hospital Clínic de Barcelona. C/Villarroel 170, Barcelona 08036, Spain. Fax: +3493 227 5783; E-mail: etolosa@clinic.ub.es and
}

Coro Paisán-Ruiz, Department of Neurology, Icahn School of Medicine at Mount Sinai, One Gustave L. Levy Place, New York, NY 10029, USA. Tel.: +1 212241 0108; Fax: +1 212828 4221; E-mail: coro.paisan-ruiz@mssm.edu. 


\section{INTRODUCTION}

In recent years genome-wide association studies (GWAS) and whole exome or genome sequencing analyses have identified common susceptibility loci associated with increased risk of Parkinson's disease (PD). The first large-scale meta-analysis of GWAS in PD nominated single nucleotide polymorphisms (SNP) in five new susceptibility loci including ACMSD, STK39, MCCC1/LAMP3, SYT11, and $C C D C 62 / \mathrm{HIP} 1 \mathrm{R}$ [1]. The association of ACMSD, aminocarboxymuconate semialdehyde decarboxylase, variants and PD has been later replicated by different groups [2-4]. A recent study has reported a rare $A C M S D$ stop codon mutation (p.Trp26Stop) in a family with autosomal-dominant cortical myoclonus, epilepsy, and parkinsonism [5]. In addition, ACMSD deficiency has been implicated in the pathogenesis of several neurodegenerative disorders [6]. Yet typical PD cases carrying an ACMSD mutation have so far not been described. Collectively, these observations make the $A C M S D$ gene an interesting candidate for genetic screening in PD but until date, no specific mutational screenings in the gene have been performed in PD populations. In this context, here we report for the first time the presence of a p.Glu298Lys mutation (rs775129424) in the ACMSD gene in a subject with PD.

\section{METHODS}

\section{Subjects}

As part of a genetic study to identify potential pathogenic gene defects related to PD in the Mediterranean island Menorca, an initial group of $62 \mathrm{PD}$ patients underwent mutational screening. PD was diagnosed according to the UK Parkinson's Disease Society criteria [7]. The study was approved by the Ethics Committee of Hospital Clínic de Barcelona and written informed consent was signed by all participants. DNA samples belonging to 192 ethnicity-matched neurologically normal individuals, without positive family history of PD, were also collected in the same time period for this study.

\section{Mutational screening}

We have used a panel-based sequencing approach to sequence more than 80 different genes known to be involved in movement disorders [8] in this population of PD patients. In our first exploratory analysis we studied the initial 62 patients. Custom libraries were generated through the HaloPlex target enrichment system (Agilent technologies, Foster City, CA, USA) following manufacturer's instructions and sequenced by using 150-bp paired-end reads on a MiSeq desktop sequencer (Illumina Inc., San Diego, CA, USA). DNA libraries $(n=12 / 13)$ were multiplexed on a single run and analyzed through GeneSifter Analyses Edition (GSAE; FinchTV 1.4.0; Geospiza Inc.; Seattle, WA, USA; http://www.geospiza.com/). All identified, genetic variants were later validated through Sanger sequencing and examined in 192 ethnicitymatched neurologically normal individuals.

To exclude normal variation, genetic variants were checked in public SNP databases, such as dbSNP149 build, the Exome Variant Server of the National Heart, Lung, and Blood Institute (NHLBI) Exome Sequencing Project (http://evs.gs.washington.edu/ EVS/), and the Genome Aggregation Database (gnomAD; http://gnomad.broadinstitute.org). The professional Human Gene Mutation (HGMD; http:// www.biobase-international.com/product/hgmd) and the NCBI ClinVar (http://www.ncbi.nlm.nih.gov/ clinvar/) databases were used to determine whether identified, genetic variants were known to be disease-causing mutations. To determine the pathogenicity of the ACMSD p.Glu298Lys mutation, the following computational prediction programs were used: MutPred (http://mutpred.mutdb.org/), SNPs\&GO (http://snps.biofold.org/), SIFT (http:// provean.jcvi.org/), and Mutation Taster (http://www. mutationtaster.org/).

\section{RESULTS}

After panel-based sequencing and Sanger sequencing validation of genetic variants identified by Haloplex targeted resequencing, we found mutations already associated with PD, and also novel mutations (manuscript in preparation). We report here one novel mutation in the $A C M S D$ gene consisting in a $\mathrm{G}$ to $\mathrm{A}$ transition and leading to the aminoacid change p.Glu298Lys, which was found in one apparently sporadic PD patient without familial history of disease at least in first degree relatives. In addition, known mutations causative of PD were not found in this patient. To exclude population normal genetic variability, we screened this mutation by Sanger sequencing in 192 ethnicity-matched neurologically normal individuals from Menorca and found that this variant was absent in these 384 control chromosomes. 
Because the mother of the PD patient carrying the $A C M S D$ mutation turned out to be of Jewish ancestry, the mutation was also tested and found to be absent in 705 Ashkenazi-Jewish genomes from the Ashkenazi Genome Consortium (https://ashkenazigenome.org). It was as well found highly conserved among other orthologous (data not shown). We did not test pathogenicity of the variant, but various computational methods predicted the p.Glu298Lys mutation to be pathogenic including MutPred (score: 0.647), SNPs\&GO (score: disease (0.596), SIFT (score: deleterious), and Mutation taster (score: disease causing). Although this mutation was found to be present in the gnomAD database, which may include some individuals with severe diseases, its frequency was very low and was identified in 2 out of 245,534 individuals tested (http://gnomad.broadinstitute.org/ variant/2-135655953-G-A), which can be compatible with pathogenicity predictions.

\section{Patient's description}

The PD patient carrying this novel ACMSD mutation was 77-years-old at the time of genetic testing. At the age of 74 years he developed right hand action tremor, speech problems and slowness. He also noticed hypersalivation and excessive daytime sleepiness. He was of Sephardic Jewish ancestry by his maternal family and of English origin by his paternal family. There was not family history of PD among his first degree relatives. He had 3 paternal uncles and one maternal uncle, all alive and healthy. He was an only child. He had two children, 43 and 39-yearsold, both healthy. At the time of presentation, he had moderate axial and appendicular rigidity, asymmetric bilateral bradykinesia, and bilateral action tremor. The UPDRS-III total score was 37.

He was started on levodopa with significant improvement. Two years later, he developed wearing off, and mild on period dyskinesias with occasional foot dystonia. The dosage of levodopa was adjusted with almost resolution of the motor complications. Rotigotine was started one year later because of worsening of symptoms. Three years after treatment onset he had a good functional state. On neurological examination dysarthria, hypomimia, and mild symmetric bilateral bradykinesia were present. Gait and postural reflexes were normal. He did not show action or rest tremor. The total MDS-UPDRS III score was 15. Other symptoms included sialorrhea, constipation and subjective olfactory loss, all starting after onset of motor symptoms. He had no symptoms suggestive of urinary dysfunction, REM sleep behavior disorder, depression, hallucinations or cognitive decline.

\section{DISCUSSION}

Here we report the first case of a subject with typical PD carrying a mutation (p.Glu298Lys) in the ACMSD gene. Although common genetic polymorphisms in the vicinity of the ACMSD gene were previously shown to be associated with increased risk for PD by several GWAS studies [1-4], we know of no reports on specific mutation in this gene in patients with sporadic PD. Recently, a stop codon $A C M S D$ mutation (p.Trp26Stop) has been reported in a family with cortical myoclonus, epilepsy, and parkinsonism [5]. In this family affected members suffered from seizures and postural hand tremor. One family member also presented, apart from seizures, parkinsonism with gait and postural abnormalities, orthostatic tremor, akinesia and rigidity of neck and arms.

The ACMSD gene encodes for an enzyme in the kynurenine pathway, aminocarboxymuconate semialdehyde decarboxylase, involved in the metabolism of tryptophan, and some metabolites of the kynurenine pathway are known to play an important role in the central nervous system in both health and disease states [9-11]. Some ACMSD mutations result in a decrease of its enzymatic activity, which leads to cellular quinolinate accumulation [12], thought to result in disturbances in synaptic plasticity and neurodegeneration [6]. There is much evidence supporting the hypothesis that the kynurenine pathway plays a critical causative role in the pathophysiology of several neurodegenerative disorders. Quinolinate selectively activates NMDA receptors, which can lead to excitotoxicity, and intrastriatal injections of this metabolite lead to axon-sparing neuronal lesions proximal to the site of injection [13, 14]. Quinolate has been also related to mitochondrial dysfunction [15]) and has been demonstrated to cause neurodegeneration in cultures of rat corticostriatal system [16]. In fact, alterations in the levels of the kynurenine pathway metabolites have been implicated in several neurodegenerative diseases, including Alzheimer's, and PD and modulation of neuroactive kynurenine metabolites has been proposed as desirable for neuroprotection [9]. ACMSD, expressed in liver, kidney and brain, has previously been related to neuropsychiatric disorders because of its crucial position in the metabolism of tryptophan degradation $[17,18]$. 
Our patient did not present any liver or kidney disease.

The mutation p.Glu298Lys involves the substitution of an acidic glutamate aminoacid, electrically charged negative, by basic Lysine which is positively charged. Although we did not perform functional analysis of the mutation, this net change of electric charges might affect protein structure and function, as predicted by pathogenicity softwares. We cannot establish a causal effect of p.Glu298Lys in ACMSD. Yet none of the known mutations causative of PD were identified in this subject. Although we cannot rule out that the mutation found in the ACMSD gene is coincidental and unrelated to the neurological syndrome it could be possible to ascribe a role for the mutation detected as a genetic risk factor, or perhaps as a rare potentially causative variant, of the patient's parkinsonism. This assumption is further supported by the identification of $A C M S D$ common genetic variability in other polymorphisms as a risk factor for PD by numerous different groups, the identification of an $A C M S D$ null mutation, not previously reported, in a family with a complex neurological phenotype including parkinsonism, and the low frequency of our reported mutation in public databases (8.146E-06). The fact that our patient carrying the p.Glu298Lys missense mutation in the $A C M S D$ gene had a milder phenotype than the family reported with the $A C M S D$ p.Trp26Stop mutation [5], a stop codon mutation, may be related to the type of mutation. Some nonsense, frameshift, or deletions in movement disorders genes, such as those in DNAJC6 and SYNJI genes, are associated with more severe phenotype than missense mutations in the same genes.

In conclusion, we report here for the first time the p.Glu298Lys mutation in the ACMSD gene in a patient with late onset idiopathic PD. This finding could point towards a potential deregulation of kynurenine pathway in some cases with this disorder. Further genetic screening in PD populations and experimental studies are warranted to elucidate the potential association of rare variants in the $A C M S D$ gene with PD.

\section{ACKNOWLEDGMENTS}

We thank The Ashkenazi Genome Consortium (https://ashkenazigenome.org/) for the genetic testing in Ashkenazi-Jewish genomes.

This study was supported by a research grant from the National Institute of Neurological Disor- ders and Stroke of the National Institutes of Health (R01NS079388; C.P.R.).

\section{CONFLICTS OF INTEREST}

The authors have no conflict of interest to report.

\section{REFERENCES}

[1] International Parkinson Disease Genomics Consortium, Nalls MA, Plagnol V, Hernandez DG, Sharma M, Sheerin UM, Saad M, Simón-Sánchez J, Schulte C, Lesage S, Sveinbjörnsdóttir S, Stefánsson K, Martinez M, Hardy J, Heutink P, Brice A, Gasser T, Singleton AB, \& Wood NW (2011) Imputation of sequence variants for identification of genetic risks for Parkinson's disease: A meta-analysis of genome-wide association studies. Lancet, 377, 641-649.

[2] Nalls MA, Pankratz N, Lill CM, Do CB, Hernandez DG, Saad M, DeStefano AL, Kara E, Bras J, Sharma M, Schulte C, Keller MF, Arepalli S, Letson C, Edsall C, Stefansson H, Liu X, Pliner H, Lee JH, Cheng R, International Parkinson's Disease Genomics Consortium (IPDGC), Parkinson's Study Group (PSG) Parkinson's Research, The Organized GENetics Initiative (PROGENI), 23 and Me; GenePD, NeuroGenetics Research Consortium (NGRC), Hussman Institute of Human Genomics (HIHG), Ashkenazi Jewish Dataset Investigator, Cohorts for Health and Aging Research in Genetic Epidemiology (CHARGE), North American Brain Expression Consortium (NABEC), United Kingdom Brain Expression Consortium (UKBEC), Greek Parkinson's Disease Consortium, Alzheimer Genetic Analysis Group, Ikram MA, Ioannidis JP, Hadjigeorgiou GM, Bis JC, Martinez M, Perlmutter JS, Goate A, Marder K, Fiske B, Sutherland M, Xiromerisiou G, Myers RH, Clark LN, Stefansson K, Hardy JA, Heutink P, Chen H, Wood NW, Houlden H, Payami H, Brice A, Scott WK, Gasser T, Bertram L, Eriksson N, Foroud T, \& Singleton AB (2014) Large-scale meta-analysis of genome-wide association data identifies six new risk loci for Parkinson's disease. Nat Genet, 46, 989-993.

[3] Pihlstrøm L1, Axelsson G, Bjørnarå KA, Dizdar N, Fardell C, Forsgren L, Holmberg B, Larsen JP, Linder J, Nissbrandt H, Tysnes OB, Ohman E, Dietrichs E, \& Toft M (2013) Supportive evidence for 11 loci from genome-wide association studies in Parkinson's disease. Neurobiol Aging, 34, 1708.e7-e13.

[4] Bandrés-Ciga S, Price TR, Barrero FJ, Escamilla-Sevilla F, Pelegrina J, Arepalli S, Hernández D, Gutiérrez B, Cervilla J, Rivera M, Rivera A, Ding JH, Vives F, Nalls M, Singleton A, \& Durán R (2016) Genome-wide assessment of Parkinson's disease in a Southern Spanish population. Neurobiol Aging, 45, 213.e3-e9.

[5] Martí-Massó JF, Bergareche A, Makarov V, Ruiz-Martinez J, Gorostidi A, López de Munain A, Poza JJ, Striano P, Buxbaum JD, \& Paisán-Ruiz C (2013) The ACMSD gene, involved in tryptophan metabolism, is mutated in a family with cortical myoclonus, epilepsy, and parkinsonism. J Mol Med (Berl), 91, 1399-1406.

[6] Stone TW, \& Darlington LG (2002) Endogenous kynurenines as targets for drug discovery and development. Nat Rev Drug Discov, 1, 609-620. 
[7] Hughes AJ, Daniel SE, Kilford L, \& Lees AJ (1992) Accuracy of clinical diagnosis of idiopathic Parkinson's disease: A clinico-pathological study of 100 cases. J Neurol Neurosurg Psychiatry, 55, 181-184.

[8] Krebs CE, \& Paisán-Ruiz C (2012) The use of nextgeneration sequencing in movement disorders. Front Genet, 3, 75 .

[9] Amaral M, Outeiro TF, Scrutton NS, \& Giorgini F (2013) The causative role and therapeutic potential of the kynurenine pathway in neurodegenerative disease. $\mathrm{J} \mathrm{Mol} \mathrm{Med,} \mathbf{9 1}$, 705-713.

[10] Vécsei L, Szalárdy L, Fülöp F, \& Toldi J (2013) Kynurenines in the CNS: Recent advances and new questions. Nat Rev Drug Discov, 12, 64-82.

[11] Schwarcz R, Bruno JP, Muchowski PJ, \& Wu HQ (2012) Kynurenines in the mammalian brain: When physiology meets pathology. Nat Rev Neurosci, 13, 465-477.

[12] Fukuwatari T, Ohsaki S, Fukuoka S, Sasaki R, \& Shibata K (2004) Phthalate esters enhance quinolinate production by inhibiting alpha-amino-beta-carboxymuconate-epsilonsemialdehyde decarboxylase (ACMSD), a key enzyme of the tryptophan pathway. Toxicol Sci, 81, 302-308.

[13] Stone TW, \& Perkins MN (1981) Quinolinic acid: A potent endogenous excitant at amino acid receptors in CNS. Eur J Pharmacol, 72, 411-412.
[14] Schwarcz R, \& Köhler C (1983) Differential vulnerability of central neurons of the rat to quinolinic acid. Neurosci Lett, 38, 85-90.

[15] Rios C, \& Santamaria A (1991) Quinolinic acid is a potent lipid peroxidant in rat brain homogenates. Neurochem Res, 16, 1139-1143.

[16] Whetsell WO Jr, \& Schwarcz R (1989) Prolonged exposure to submicromolar concentrations of quinolinic acid causes excitotoxic damage in organotypic cultures of rat corticostriatal system. Neurosci Lett, 97, 271-275.

[17] Brundin L, Sellgren CM, Lim CK, Grit J, Pålsson E, Landén M, Samuelsson M, Lundgren K, Brundin P, Fuchs D, Postolache TT, Traskman-Bendz L, Guillemin GJ, \& Erhardt S (2016) An enzyme in the kynurenine pathway that governs vulnerability to suicidal behavior by regulating excitotoxicity and neuroinflammation. Transl Psychiatry, 6, e865.

[18] Garavaglia S, Perozzi S, Galeazzi L, Raffaelli N, \& Rizzi M (2009) The crystal structure of human $\alpha$ amino- $\beta$-carboxymuconate- $\varepsilon$-semialdehyde decarboxylase in complex with 1,3-dihydroxyacetonephosphate suggests a regulatory link between NAD synthesis and glycolysis. FEBS J, 276, 6615-6623. 